\title{
NORM EXPONENTS AND REPRESENTATION GROUPS
}

\author{
HANS OPOLKA
}

(Communicated by William Adams)

\begin{abstract}
This note provides an upper bound for the exponent of the norm residue group $k^{*} / \operatorname{Norm}_{K / k}\left(K^{*}\right)$ of a finite Galois extension $K / k$ of number fields that depends on the obstruction to the Hasse norm principle for $K / k$ and on a group theoretical constant.
\end{abstract}

Let $K / k$ be a finite Galois extension of number fields with Galois group $G=\operatorname{Gal}(K / k)$. We call

$$
\nu=\nu(K / k)=\text { exponent } \text { of } k^{*} / \operatorname{Norm}_{K / k}\left(K^{*}\right)
$$

the norm exponent of $K / k$. Obviously $\nu$ divides the degree $(K: k)$, and from the density theorem and the local reciprocity isomorphism we see that $\exp (G)$ divides $\nu$. In this note we derive a "good" upper bound for $\nu$ which depends on the obstruction to the Hasse norm principle for $K / k$, i.e. on the kernel $\mathscr{K}=\mathscr{K}(K / k)$ of the natural map

$$
\widehat{H}^{0}\left(G, K^{*}\right) \rightarrow \widehat{H}^{0}\left(G, \mathbf{A}_{K}^{*}\right),
$$

where $\widehat{H}^{0}$ denotes the Tate cohomology in dimension 0 and $\mathbf{A}_{K}^{*}$ the group of units of the adele ring $\mathbf{A}_{K}$ of $K$, and on a group theoretical constant. It implies and improves all previous results in this respect $[4 ; 7 ; 8, \mathrm{p} .100]$. Tate has observed (see [2, p. 198]) that $\mathscr{K}$ is dual to the kernel $\mathscr{H}=\mathscr{H}(K / k)$ of the localization map

$$
H^{2}\left(G, \mathbf{C}^{*}\right) \rightarrow \coprod_{v} H^{2}\left(G_{\bar{v}}, \mathbf{C}^{*}\right)
$$

here $G_{\bar{v}}$ denotes the decomposition group of an extension $\bar{v}$ of the place $v$ of $k$ and cohomology is taken with respect to the trivial group action. A finite group extension $\widetilde{G}$ of $G$ is said to be defined by a subgroup $\mathscr{A} \leq H^{2}\left(G, \mathbf{C}^{*}\right)$ if $\mathscr{A}$ is contained in the kernel of the inflation map $H^{2}\left(G, \mathbf{C}^{*}\right) \rightarrow H^{2}\left(\widetilde{G}, \mathbf{C}^{*}\right)$. Define

$$
\lambda=\lambda(K / k)=\text { minimum of all } \exp (\widetilde{G}),
$$

Received by the editors October 20, 1988 and, in revised form, January 20, 1990.

1980 Mathematics Subject Classification (1985 Revision). Primary 12A10, 12 A65. 
where $\widetilde{G}$ runs over all finite group extensions of $G$ which are defined by $\mathscr{H}$. (Note that, in contrast to $[7$, lines $17 / 18]$ it is not required that the embedding problem corresponding to $\widetilde{G}$ is solvable.) For any natural number $r$ put

$$
X(k, r):=\bigcap_{v}\left(k^{*} \cap\left(k_{v}^{*}\right)^{r}\right) / k^{* r},
$$

where $v$ runs over all places of $k$; it is well known that $X(k, r)$ is trivial if $r$ is odd and that $|X(k, r)| \leq 2$ in any case (see, e.g. [1, p. 93ff]). We prove

1. Theorem. $\nu$ divides $\lambda \cdot|X(k, \lambda)|$.

Proof. Represent every cocycle class $(f) \in \mathscr{H}$ by a cocycle $f: G \times G \rightarrow \mu_{m}$, $\mu_{m}=$ group of roots of unity in $\mathbf{C}^{*}$ of order $m=\exp (\mathscr{H})$, such that the central group extension $G(f)$ defined by $(f)$ has minimal exponent. Put $\tilde{m}=\tilde{m}_{f}=$ $n \cdot|X(k, n)|$ where $n=n_{f}=\exp (G(f))$. Let $C_{r}$ be the cyclic group of order $r$. We assume that the action of $G$ on $C_{r}$ is trivial. $(f) \in \mathscr{H}$ implies that the class of the induced cocycle

$$
f^{\prime}: G \times G \rightarrow C_{m} \hookrightarrow C_{n}
$$

(we identify $C_{r}$ with $\mu_{r}$ ) belongs to the kernel of the homomorphism

$$
H^{2}\left(G, C_{n}\right) \rightarrow \prod_{v} H^{2}\left(G_{\bar{v}}, C_{n}\right) .
$$

Let $X_{r}$ be the kernel of the homomorphism

$$
H^{2}\left(G_{k}, C_{r}\right) \rightarrow \prod_{v} H^{2}\left(G_{k_{v}}, C_{r}\right),
$$

where $G_{k}$ resp. $G_{k_{v}}$ are the absolute Galois groups of $k$ and $k_{v}$ respectively. $X_{r}$ is dual to $X(k, r)$. It follows that $\operatorname{Inf}_{G_{k}}\left(\left(f^{\prime}\right)\right) \in X_{n}$. Since the natural map $X(k, \tilde{m}) \rightarrow X(k, n)$ is trivial, it follows that the canonical homomorphism $X_{n} \rightarrow X_{\tilde{m}}$ is trivial. Hence the embedding problem defined by the cocycle

$$
f_{1}: G \times G \rightarrow \mu_{m} \hookrightarrow \mu_{\tilde{m}}
$$

has a surjective solution (see, e.g., [3, especially pp. 88, 96]). Let $L_{f_{1}} / K / k$ be a solution of this embedding problem and denote by $L$ the compositum of all $L_{f_{1}},(f) \in \mathscr{H}$. Then $\operatorname{Gal}(L / k)$ is defined by $\mathscr{H}$. As shown in [6, (2.5)], this means that every element in $k^{*}$ which is a norm locally everywhere in $L / k$ is a global norm in $K / k$. As remarked earlier the density theorem and local class field theory show that $\exp (\operatorname{Gal}(L / k))$ is the l.c.m. of all the local norm exponents of $L / k$. Hence $\nu(K / k)$ divides $e=\exp (\operatorname{Gal}(L / k))$. The equality $\exp \left(\operatorname{Gal}\left(L_{f_{1}} / k\right)\right)=\tilde{m}_{f}$ shows that $e$ divides the l.c.m. of all $\tilde{m}_{f},(f) \in \mathscr{H}$. Clearly $\widetilde{G}:=\times_{(f)} G\left(f_{1}\right),(f) \in \mathscr{H}$, is defined by $\mathscr{H}$, and the exponent of $\widetilde{G}$ equals $\lambda \cdot|X(k, \lambda)|$. This completes the proof. 
2. Remark. Clearly $\nu(K / k)$ divides the 1.c.m. of all $\nu\left(K / k^{p}\right), p$ a prime, where $k^{p}$ is the fixed field of a $p$-Sylow subgroup $G^{p}$ of $G$, because the restriction map $\widehat{H}^{0}\left(G, K^{*}\right) \rightarrow \widehat{H}^{0}\left(G^{p}, K^{*}\right)$ is injective on the $p$-part of $\widehat{H}^{0}\left(G, K^{*}\right)$. For any finite group $G$ define

$$
\delta(G):= \begin{cases}1 & \text { if }|G| \text { is odd }, \\ 2 & \text { if }|G| \text { is even. }\end{cases}
$$

Every finite abelian group $G$ has a representation group $\widetilde{G}$ such that $\exp (G)$ divides $\delta(G) \cdot \exp (G)$. This comes from the isomorphism $H^{2}\left(G, \mathrm{C}^{*}\right) \cong(G \wedge G)^{\wedge}$ given by $(f) \mapsto \omega_{(f)}$ where

$$
\omega_{(f)}(x, y)=f(x, y) / f(y, x), \quad x, y \in G .
$$

Since $\omega_{(f)}$ is a symplectic pairing on $G$ we may choose the cocycle $f$ in such a way that $f$ is bimultiplicative. Then an easy computation shows that the group extension defined by $f$ has exponent dividing $\delta(G) \cdot \exp (G)$.

In $[9$, Corollary (4.7)] it is shown that every $p$-group $G, p \neq 2$, such that the class of $G$ is $\leq p-2$ and such that $\exp (G)=p$ has a representation group of exponent $p$.

Furthermore, by [5, V, 24.5], the exponent of any representation group of a finite group $G$ divides the order of $G$.

Therefore 1 and 2 give the following result

3. Proposition. Assume that every $p$-Sylow subgroup of the finite Galois group $G=\operatorname{Gal}(K / k)$ is abelian or of exponent $p \neq 2$ and class $\leq p-2$. Then $\nu$ divides $\delta(G) \cdot|X(k, r)| \cdot \exp (G)$ where $r=|G|=(K: k)$.

\section{ACKNOWLEDGMENT}

I would like to thank the referees for their remarks.

\section{REFERENCES}

1. E. Artin and J. Tate, Class field theory, Benjamin, New York, 1967.

2. J.W.S. Cassels and A. Fröhlich, Algebraic number theory, Academic Press, New York, 1967.

3. K. Hoechsmann, Zum Einbettungsproblem, J. Reine Angew. Math. 229 (1968), 81-106.

4. W. Hürlimann and D. Saltman, On the exponent of norm residue groups, Proc. Amer. Math. Soc. 93 (1985), 417-419.

5. B. Huppert, Endliche Gruppen I, Springer-Verlag, Berlin, 1967.

6. H. Opolka, Zur Auflösung zahlentheoretischer Knoten, Math. Z. 173 (1980), 95-103.

7. (1987), 41-43.

8. G. Steinke, Über Auflösungen zahlentheoretischer Knoten, Schriftenreihe Math. Inst. Univ. Münster, Ser. 2, Heft 25, 1982.

9. H. Suzuki, On a central solution of the number knot of a finite p-extension of nilpotent class $\leq p-1$, preprint, 1988 . 